\title{
Géotechnique et archéologie
}

\author{
Geotechnique and archeology
}

\author{
Jean KERISEL
}

Rev. Franç. Géotech., n 63, pp. $5 \cdot 7$ (avril 1993)

Les congrès et conférences internationales sont fréquemment l'occasion de se féliciter du travail accompli et des progrès enregistrés. Et si un historien n'avait à sa disposition que les conclusions écrites de symposiums, il affirmerait, bien à tort, que sciences et techniques n'ont connu qu'une ascension continue.

L'archéologie des grands édifices projette parfois une lumière intéressante sur les premiers chemins de la connaissance en apportant la preuve de toute une suite d'échecs et de succès.

Pour le montrer, je vais prendre des exemples en Egypte et en Mésopotamie. L'attention sera centrée sur quatre domaines: les fondations en surface, les soutènements, les frottements négatifs et les ouvrages souterrains.

\section{FONDATIONS SUPERFICIELLES ET SOUTENEMENTS}

\subsection{L'Egypte}

Toutes les grandes pyramides d'Egypte ont été érigées dans un intervalle de temps de l'ordre de cinq cents ans seulement pendant la première partie de l'Ancien Empire: elles furent érigées toutes sur la rive ouest du Nil.

La toute première, Saqqarah, pyramide à cinq gradins à une trentaine de kilomètres au sud de l'actuelle ville du Caire, est bâtie sur un sol rocheux et par conséquent n'a pas posé de problème de fondations superficielles. Elle est par ailleurs l'illustration de la parfaite compréhension par les Egyptiens des problèmes de murs de soutènement. A cette époque, vers 2700 av. J.-C., pour tailler des pierres on ne disposait que de moyens très limités (frottement par de la dolérite et scies au cuivre durcies par de l'émeri. Il n'était donc pas question de tailler toutes les pierres d'une pyramide de $60 \mathrm{~m}$ de haut. Il fallait s'efforcer en outre de réutiliser les volu- mineux déchets de pierres résultant de la taille. Or, ces déchets, lorsqu'ils sont superposés, poussent au vide et cette poussée demande à être contenue par des murs de soutènement. Imhotep, le ministre-architecte du pharaon, imagina de ne tailler que les pierres destinées à construire des murs de soutènement, inclinés à $72^{\circ}$ sur l'horizontale vers l'intérieur de la pyramide, et d'intercaler entre les murs distants de $2,50 \mathrm{~m}$ les uns des autres, les déchets de pierre. Ainsi était économisé l'effort humain, tout en assurant une parfaite stabilité. Les parties supérieures des murs de soutènement sont les contremarches des gradins de la pyramide, l'enveloppe de l'ensemble formant un tétraède dont les faces sont inclinées à $52^{\circ}$ sur l'horizontale.

La grande pyramide suivante fut celle de Meidoum à $70 \mathrm{~km}$ au sud du Caire. Elle fut d'abord une pyramide à gradins d'une soixantaine de mètres de hauteur conçue comme celle de Saqqarah. On emboîta dessus une autre pyramide à gradins dont la hauteur atteignît $70 \mathrm{~m}$. Enfin le pharaon suivant la transforma en pyramide lisse dont les quatre faces enveloppent les gradins de la deuxième pyramide à gradins par l'intermédiaire d'un matériau de mauvaise qualité, trop friable, si bien que, celui-ci cédant peu à peu aux cisaillements qui le sollicitaient sur une aussi forte pente ( $52^{\circ}$ en moyenne), il se produisit un grand glissement sur chacune des quatre faces de la pyramide, mettant à nu sur une grande hauteur $(30 \mathrm{~m})$ l'un des murs intérieurs de soutènement.

La pyramide suivante est celle de Dahchour: son emplacement avait été choisi par le pharaon Snefrou, très entreprenant mais moins bon géologue puisqu'il résolut de l'implanter dans le lit majeur du Nil sur des formations relativement récentes ayant seulement la résistance d'argiles raides. Chaque pharaon essayait alors de construire sa demeure d'éternité plus haute que celle de son prédécesseur et même plus élancée. L'ambition de Snefrou alla jusqu'à essayer de construire sa pyramide avec des versants très raides inclinés sur la verticale à pas moins de $60^{\circ}$ alors que ses prédécesseurs s'en étaient tenus à $52^{\circ}$. Sa pyramide devait ainsi culminer à $140 \mathrm{~m}$ de hauteur. Très rapidement le sol fut poinçonné et il fut obligé d'élargir la base en diminuant 
l'angle d'inclinaison de $60^{\circ}$ à $54^{\circ} 31$. L'élargissement céda à son tour comme le prouvent les ressauts que l'on constate dans les couloirs qui descendent aux chambres funéraires et Snefrou n'eut d'autre ressource, arrivé à $43 \mathrm{~m}$ de haut que de diminuer à nouveau la pente de $54^{\circ} 31$ à $43^{\circ} 5$ d'où le nom de rhomboïdale donné à cette pyramide, qui est l'illustration type d'un monument sur une fondation qui se dérobe sous l'effet d'une trop forte charge à l'intérieur d'un périmètre donné.

Les pyramides suivantes, les dernières grandes pyramides de l'Ancien Empire, qui constituent la triade de Gizeh, ne connurent aucun incident de fondation. Ces pyramides sont celles de Kheops, Khefren et Mykérinos, fondées sur le calcaire éocène du plateau de Gizeh à l'ouest de la ville du Caire actuelle. Kheops et Khefren ont une hauteur qui avoisine $150 \mathrm{~m}$ et avec un poids spécifique moyen de 2 tonnes par $\mathrm{m}^{3}$, elles apportent une pression au sol importante. Kheops, lui, était fin géotechnicien et il avait choisi la zone du plateau où la résistance du rocher est la meilleure; mieux même, il traita les quelques failles du plateau sur la pyramide en y enfonçant au marteau, à force, des coins de pierre taillée.

\subsection{La Mésopotamie}

Bien avant les Egyptiens, les Sumériens qui habitaient le Sud de la Mésopotamie à Our, Ourouk, Eridou, dans la zone à l'Ouest de Bassorah, dans ce delta où aboutissent ces deux fleuves puissants, l'Euphrate et le Tigre, avaient tenté d'ériger, eux aussi, des monuments à forme tronc-pyramidale, Mais pour eux dans ce delta marécageux où le sol est de faible portance, l'entreprise était malaisée. Ne disposant pas de pierres, ils construisaient ces monuments appelés ziggourats, avec des briques faites de limon séché au soleil. Ils ne tardèrent pas à s'enfoncer dans le sol et à s'écarteler faute de résistance au poinçonnement et au cisaillement latéral. Ils ne purent élever ce genre de monument qu'à partir du moment où ils intercalèrent, tous les cinq ou six lits de briques, des tapis en roseaux tissés et même des câbles faits de végétaux. De cette façon, ils évitèrent l'écartèlement et le bâtiment après avoir trouvé un équilibre en profondeur put monter vers le ciel. Il s'agit d'une longue étude empirique de fondations superficielles sur terrains mous.

La civilisation se développa vers le nord: les cités devinrent des Cités-Etats. Chacune avait une ziggourat et la hauteur de la ziggourat témoignait de la puissance de la Cité-Etat. Bientôt des royaumes apparurent: l'un des rois les plus puissants fut celui de Babylone vers 1800 av. J.-C. : Hammurabi. Il veillait à la stabilité des constructions, puisque son code, que beaucoup d'ingénieurs connaissent, témoigne, par sa sévérité, de sa volonté d'éviter les premiers accidents des ziggourats.

La ziggourat qu'il avait construite fut saccagée par Sennacherib, roi d'Assyrie (705-680 av. J.-C.). Elle fut reconstruite et élargie, par Nabuchodonosor II le grand roi de la deuxième Babylone vers 600 av. J.C. Cette nouvelle ziggourat n'est autre que la tour de Babel. Son empreinte au sol qui mesure $91 \times 91 \mathrm{~m}$ montre bien que la partie centrale se trouvait sur une zone déjà consolidée par la ziggourat de la première Babylone alors que l'élargissement sur terrain vierge en bordure de l'Euphrate, a marqué profondément le sol de son empreinte.

Un deuxième incident de fondations survint dans cette deuxième Babylone: celui de la porte d'Ishtar, une porte en briques cuites, très lourde, revêtue de belles briques émaillées bleu foncé avec des dessins d'animaux. Nabuchodonosor savait sans doute qu'il lui fallait prendre des précautions. Aussi il la bâtit sur un remblai de sable qu'il épandit sur quelques mètres de hauteur au-dessus du sol limoneux. Précaution qui se révéla absolument insuffisante. Les massifs de briques, des deux côtés de la porte, poinçonnèrent le sable et descendirent dans le limon sur une hauteur de quinze mètres comme l'a montré l'archéologue allemand KOL. DEWEY au début de ce siècle.

\section{FROTTEMENT NÉGATIF OUVRAGES SOUTERRAINS}

Je voudrais m'intéresser maintenant aux fondations profondes ou plus exactement au frottement qui se développe sur le bord des fondations profondes.

Comme on le sait, dans une fondation profonde faite dans un milieu assez homogène, la résistance du sol sous la base, tout autant que le frottement qu'il exerce latéralement à la fondation de bas en haut, contribuent à porter les charges. Ce dernier frottement est appelé positif.

Mais il arrive aussi que ce frottement s'inverse et devienne négatif, s'ajoutant aux charges de la fondation: c'est le cas lorsque la fondation s'appuie sur un terrain dur dominé par un terrain en cours de consolidation. L'attention du constructeur doit toujours rester en éveil dans ce cas de frottement négatif. Pour avoir mésestimé ce frottement négatif, la fin de la construction de la pyramide de Kheops a rencontré de sérieuses difficultés. La pyramide comporte en effet une dualité de maçonnerie. La première en pierres de taille parfaitement assisées concerne la carapace et un socle interne, étroit, orienté Nord-Sud qui contient les corridors, la grande galerie et deux chambres dont la plus vaste est celle dite chambre du Roi. La seconde maçonnerie entoure le socle, réalisée en opus incertum à partir de pierres calcaires de qualité moyenne. Elle est évidemment plus compressible que la première. Et sous l'effet de la pression énorme qui résulte d'une hauteur de $150 \mathrm{~m}$, elle a tassé.

La chambre du roi n'avait pas moins de $5,90 \mathrm{~m}$ de haut et une section horizontale de $5 \times 10,5 \mathrm{~m}$. Dimensions considérables au regard des $100 \mathrm{~m}$ de maçonnerie qui la surchargent. Elle est couverte par neuf grandes dalles de granit (de $2 \mathrm{~m}$ d'épaisseur) alignées côte à côte dans le sens du petit côté. Kheops pressentant sans doute qu'elles pourraient fléchir et casser, avait imaginé de superposer cinq autres chambres à sa chambre. Les archéologues ont lhabitude d'appeler ces chambres, chambres de décharge. Elles sont au contraire des chambres surchargées car sous le poids 
considérable des $100 \mathrm{~m}$ de maçonnerie, la maçonnerie en opus incertum tout autour de cette chambre s'est tassée de $15 \mathrm{~cm}$ par rapport au socle et elle a formé un point dur. De plus, en descendant la maçonnerie en opus incertum a exercé sur le flanc sud de la chambre du Roi un frottement négatif amenant une distorsion de la chambre du Roi, le mur Sud de celleci devenant plus bas que le mur Nord, la différence étant de $6 \mathrm{~cm}$ au milieu du mur Sud. Or toutes les dalles en granit du toit de la chambre du Roi et des chambres supérieures étaient encastrées à leurs appuis du fait de la pression considérable s'exerçant sur ceux. ci. Une dalle de granit encastrée de deux mètres d'épais. seur et de cinq mètres de portée libre, ne peut absolument pas supporter une telle dénivellation d'appui. Toutes les dalles se sont progressivement fissurées et rompues au fur et à mesure de la fin de la construction de la pyramide. Et comme le veut la théorie des dalles encastrées, on trouve les fissures au-dessous de la dalle, du côté Sud qui a tassé, et au-dessus du côté opposé.

La rupture de ces dalles a certainement causé effroi et désespoir chez le pharaon. Il a certainement voulu connaître la raison des détonations qui en résulteraient. Après avoir déblayé les couches supérieures, il a replâtré certaines fissures: on trouve en effet la preuve de plusieurs replâtrages.

Nous savons bien, nous, ingénieurs, combien il est difficile d'insérer une structure rigide dans un remblai de main d'homme qui est moins rigide. Par exemple, il est dangereux de construire un ouvrage d'écoulement des eaux au sein même de l'ouvrage qui va barrer leur écoulement: ou bien on réalise un ouvrage trop rigide puissamment armé ou bien on introduit une série de grandes buses métalliques trop flexibles. Et ceci en plein empirisme.

La preuve des difficultés rencontrées par les Egyptiens résulte de l'évolution de leurs chambres funéraires. Au départ on renonce à insérer la chambre dans la pyramide, c'est le cas à Saqqarah où la chambre est sous la pyramide, dans le rocher à trente mètres de profondeur. Excellente solution.

La chambre à Meidoun est insérée dans la puramide au ras du sol, solution encore admissible tant que les dimensions restent modestes.

En tout cas la plus mauvaise des solutions est celle qui a consisté à Dahchour à faire deux chambres à deux niveaux différents, l'une sous la pyramide, l'autre engagée dans la pyramide. Pour faire la première, il a fallu en effet faire une fouille blindée dans cette argile raide dont nous avons parlé, construire la maçonnerie de la chambre, puis remblayer au-dessus. Lorsque l'on a commencé à élever la pyramide, des pressions trop im. portantes se sont exercées latéralement sur cette chambre qui s'est fissurée. Snefrou a alors décidé d'en construire une seconde insérée dans la pyramide. Mais alors le remblai au-dessus de la première chambre, mal compacté, a tassé amenant des désordres dans la deuxième chambre.

Cette leçon consistant à ne pas exécuter des ouvrages souterrains voisins à des niveaux différents $a$, en règle générale, été retenue en mécanique des sols.

\section{CONCLUSION}

Le progrès s'est fait de la médiation d'échecs. Apprenons à bien connaître ces échecs et retenons-en les leçons. Intéressons-nous aussi à l'archéologie où l'ap. port des ingénieurs, si rare jusqu'à présent, peut ap. porter des compléments intéressants à l'histoire des sciences et techniques. 